\title{
Essais
}

ESSAIS

Revue interdisciplinaire d'Humanités

Hors-série 6 | 2021

Agrobiodiversité et territoires

\section{Retour d'expérience d'un projet de conservatoire du goût des espèces et variétés maraîchères en métropole Bordelaise}

Rencontre avec Rachel Lagière

Feedback from a project for a taste conservatory of market garden species and varieties in Bordeaux

Meeting with Rachel Lagière

\section{OpenEdition}

\section{Édition électronique}

URL : https://journals.openedition.org/essais/7954

DOI : 10.4000/essais.7954

ISSN : 2276-0970

Éditeur

École doctorale Montaigne Humanités

Édition imprimée

Date de publication : 1 mars 2021

Pagination : 177-180

ISBN : 978-2-492780-00-4

ISSN : $2417-4211$

Référence électronique

"Retour d'expérience d'un projet de conservatoire du goût des espèces et variétés maraîchères en métropole Bordelaise », Essais [En ligne], Hors-série 6 | 2021, mis en ligne le 16 mars 2021, consulté le 18 janvier 2023. URL : http://journals.openedition.org/essais/7954 ; DOI : https://doi.org/10.4000/ essais.7954 


\section{Retour d'expérience d'un projet de conservatoire du goût des espèces et variétés maraîchères en métropole Bordelaise}

\section{Entretien avec Rachel Lagière ${ }^{1}$}

Rachel Lagière est ingénieure agronome et maraîchère, co-Fondatrice et responsable du Conservatoire du Goût à Floirac (33), spécialiste des plantes maraîchères.

\section{Pouvez-vous nous présenter le Conservatoire du Goût, avec un point rapide sur l'historique et le contexte de création ?}

Le Conservatoire du Goût est une association loi 1901, créée en septembre 2017 dans le but de sauvegarder la biodiversité cultivée ayant un intérêt gustatif, de la diffuser en encourageant des producteurs à les cultiver, ce qui participera à leur sauvegarde et à la sauvegarde du métier de paysan. Le constat est simple : nous assistons à une disparition catastrophique de la biodiversité cultivée ; les légumes, donc les plantes cultivées, semblent encore plus menacées que les races d'animaux car ils intéressent très peu malgré l'essor du véganisme et du végétarisme. La France est en retard car on a de bons produits animaux qui captent plus l'intérêt.

Ce projet est né sur une ferme maraîchère bretonne grâce à Christophe Collini, qui s'était rendu compte du faible choix en France de bons légumes et de bonnes semences. Il a très vite travaillé avec des chefs qui l'ont aidé à sélectionner et identifier de bonnes variétés. Après 5 ans, nous nous sommes rencontrés par hasard car je créais alors de mon côté une association de jardins partagés, un lieu collectif dédié à la culture de produits qui avaient du goût. Notre passion commune du goût et du partage nous a rapproché, et il m'a ainsi demandé de le rejoindre. Pendant deux ans, j’ai appris le maraîchage à ses côtés, et nous avons créé le Conservatoire du Goût, en Bretagne. Ce conservatoire, c'était une synergie de restaurateurs, de paysans, de semenciers, d'associations, de scientifiques, de passionnés de goût de légumes... Suite à

1 Dans le cadre de sa participation au séminaire des 14 et 15 mai 2019. 
cette création en septembre 2017, je suis arrivée en Gironde en 2018 pour poursuivre l'aventure. Je suis en train de créer le premier site expérimental et productif à Floirac, au nord de Bordeaux, sur un grand domaine de 70 hectares dont 50 en forêt, 20 en écurie ; milieu assez privilégié, qui reste dans l'agglomération bordelaise. Ce projet est une réponse à la manifestation d'intérêt qu'avait éditée la mairie de Floirac pour installer un maraîcher sur $5000 \mathrm{~m}^{2}$ mis en location, avec une mutualisation de serres municipales de $600 \mathrm{~m}^{2}$ dans lesquelles siégeait la floriculture pour Bordeaux Métropole depuis délocalisée au Haillan. Aujourd'hui j'utilise $50 \mathrm{~m}^{2}$ dans cette serre chauffée, ce qui permet de faire tous les semis.

\section{Quelles sont les étapes de la chaîne de production de ces légumes?}

La ferme, dans le cadre du Conservatoire du Goût, fait tous les semis sauf pour les plantes qui se bouturent ou qui se greffent (certains aromates et arbres fruitiers). Ils germent sur place puis sont repiqués en pleine terre pour grandir puis pour être récoltés et vendus. Tout se fait, pour le moment, avec un travail superficiel du sol qui est extrêmement important ; on utilise une herse rotative qui ne va pas mélanger les horizons du sol pour déranger le moins possible la pédofaune dans cette ancienne prairie. C'est une terre très riche, avec beaucoup de vers et de champignons ; elle sent bon, c'est sain ! Les serres font un peu moins de $800 \mathrm{~m}^{2}$ en pleine terre, le reste est en plein champ.

\section{Quel est à peu près le volume de variétés représentées ?}

Il y a une très grande diversité cultivée chaque année. Depuis le tout début des recherches officieuses du conservatoire, avant la création de l'association, nous avons testé au minimum 3000 à 3500 variétés de légumes toutes espèces confondues, soit une sélection de 200-300 variétés cultivées par an. Mais nous sommes encore dans une phase de test, le temps de vérifier comment se comportent les plantes.

\section{Comment se traduit la recherche des semences et du patrimoine génétique?}

Cette année par exemple, nous cultivons plus de 150 variétés de tomates, sur une base de variétés déjà sectionnées sur lesquelles sont prélevées les semences. Mais nous testons parallèlement de nouvelles variétés qui doivent être étudiées. Soit c'est une variété rare, ancienne ou nouvelle, trouvée chez un semencier n'importe où dans le monde, soit c'est une variété connue mais pour laquelle nous essayons de trouver des semences de bonne souche qui correspondent aux critères que nous recherchons en priorité, le goût (exemples de la tomate Marmande ou de la Rose de Berne). En France, nous avons finalement très peu de choix et le travail sur le goût est rare ; nous sommes très pauvres en variétés à qualités gustatives, même chez les artisans semenciers. Nous allons donc chercher des variétés principalement aux États-Unis, en Allemagne, au Japon 
ou en Angleterre. Ensuite, le travail est le même, que ce soit pour une variété qui présente un potentiel ou pour une variété commune dont il faut trouver la souche : nous sélectionnons un maximum de semenciers qui proposent la variété, et nous testons les plants jusqu'à trouver la bonne souche selon les critères de goût et de qualités nutritives. Car, quand je parle du goût, je parle de la nutrition ; pour moi, c'est évident que si un produit a du goût, c'est qu'il est nutritif. C'est un ressenti, maintenant il faudra réaliser des analyses.

\section{Comment valorisez-vous ces variétés sur les territoires de production, avec quels réseaux ?}

La sélection des variétés pour leurs qualités gustatives ne pourrait se faire sans le réseau des chefs cuisiniers. Nous organisons, tout au long du calendrier de maturité et de cueillette, des séances de dégustation avec les chefs, qui vont nous orienter sur le choix des variétés à développer et à cultiver ; une partie des chefs est originaire de la région, avec un réseau qui est en train de se développer ; l'autre partie du réseau est bretonne et parisienne. La gastronomie et l'image des chefs jouent un énorme rôle sur la promotion du travail de sélection et de sauvegarde, aujourd'hui non négligeable à la lumière de l'ampleur de la disparition des semences. Nous en profitons directement, car ce sont eux qui sont les spécialistes, avec des palais développés et éduqués capables de caractériser les goûts et les émotions suscitées par les produits. Nous travaillons avec les chefs à développer un vocabulaire qui se rapproche de l'œnologie, en créant des référentiels pour caractériser un goût. Cette clientèle de restaurateurs permet la sécurité financière et la durabilité de notre modèle économique, dans un retour constant sur les manières d'exploiter nos productions : par exemple, si une moutarde japonaise est très bonne en feuille, lorsqu' elle monte en fleur elle perd certaines qualités, elle peut devenir plus piquante, moins subtile et moins tendre et serait donc, à priori, non valorisable ; or, grâce aux chefs, on sait que la tige peut alors être utilisée car elle a un goût délicieux et doux de moutarde, une texture croquante et juteuse comme un concombre... on peut donc continuer de la valoriser. Le temps de la découverte et de l'expérimentation permet de trouver des solutions pour valoriser la plante et donc pour la développer.

\section{Justement, comment se déroule l'étape suivante, le développement agricole des variétés?}

Une fois qu'une variété est sélectionnée par les chefs, nous la faisons reproduire par les paysans multiplicateurs, avec un contrat avec les semenciers qui vont ensuite commercialiser les semences. Tout ce réseau fonctionne avec un cahier des charges Conservatoire qui est en train d'être écrit. Cela permettra aux maraîchers et aux jardiniers amateurs de pouvoir choisir des semences labellisées "Conservatoire du Goût », avec une description des qualités agronomiques et gustatives. Il faudra aussi que le maraîcher puisse savoir si cette variété a une commercialisation de niche (pas très productive mais excep- 
tionnelle sur le plan gustatif) ou si elle s'adapte bien à du maraîchage plus commun. Tout au long du processus, il y a un contrôle des qualités gustatives, qui peuvent se perdre par exemple s'il y a trop d'arrosage. Cela permet de revoir les pratiques si besoin, avec une façon de travailler qui s'adapte aux variétés grâce à la sensibilité du paysan et à l'expérimentation.

Le Conservatoire du Goût accompagne ainsi les maraîchers, dans leurs pratiques et dans leurs choix variétaux, mais également sur les débouchés et la construction du modèle économique où leur production est vendue, en partie aux chefs et en partie aux particuliers. Nous valorisons le travail des paysans lorsque les clients donnent de bons retours, car l'encouragement moral est tout aussi important que l'encouragement financier. Nous accompagnons également les restaurateurs qui veulent avoir leur potager, car c'est toujours pour nous une occasion de valoriser ces variétés. Nous avons une forte demande de retours d'expériences sur les variétés exploitables car le travail sur le goût est encore trop rare ; c'est comme cela que le réseau s'agrandit. C'est des métiers durs mais passionnants, nourrissants, parce que l'on découvre constamment, on s'adapte, on se remet en question...

\section{Comment ces actions contribuent-elle à une gestion environnemen- tale durable des territoires?}

Le travail de sauvegarde des semences est un travail de sauvegarde du patrimoine génétique, où l'on ne se limite pas à sauvegarder des espèces, mais bien toutes les variabilités intraspécifiques représentées par les variétés, accompagnées des pratiques culturales qui leur correspondent. En inhumant des variétés oubliées, on remet en lumière un patrimoine culturel lui aussi tombé en désuétude. L'entrée par le gustatif permet, en plus, d'intégrer à ces collections des espèces de plantes sauvages ou même utilisées à la base pour leurs qualités ornementales, mais qui s’avèrent délicieuses. On élargit ainsi la banque de semences qui représentent un patrimoine biologique et culturel infini.

Les pratiques culturales que nous valorisons, d'agriculture biologique ou d'agroforesterie, ont également des effets très positifs sur les écosystèmes; elles favorisent les insectes pollinisateurs, la pédofaune, tous les réseaux trophiques, tout en étant économiquement viables. On a eu par exemple le retour d'un apiculteur breton qui, en comparant les miels de ses différents sites de production, a certifié que le meilleur miel, le plus riche et explosif sur le plan gustatif, était celui produit par les abeilles qui butinaient les fleurs de nos productions. La diversité est une richesse pour le miel!

On communique énormément auprès de nos publics et de notre réseau. On fait passer un message simple, pédagogique, en prenant le temps d'expliquer que le maraîchage est un acte humaniste où il faut tout considérer, et tout faire en conscience. 